\title{
Poluição do ar e sua relação com a epilepsia
}

\author{
Air pollution and its relationship to epilepsy
}

\section{La contaminación del aire y su relación con la epilepsia}

\author{
Constanza Rocha Jaures ${ }^{1}$, Danielle dos Santos Maia Salheb de \\ Oliveira², Marcia Regina Cominetti ${ }^{2}$, Maria José da Silva Fernandes ${ }^{1}$
}

1.Universidade Federal de São Paulo, Departamento de Neurologia e Neurocirurgia, Disciplina de Neurociência, São Paulo-SP, Brasil.

2.Universidade Federal de São Carlos, Departamento de Gerontologia, São Carlos-SP, Brasil.

\begin{abstract}
Resumo
Introdução. A epilepsia é considerada uma doença neurológica que afeta mais de 50 milhões de pessoas no mundo. É caracterizada pela manifestação de crises epilépticas que são eventos com atividade transitória, autolimitada, com sinais e sintomas definidos, podendo se expressar de variadas formas a depender da área de início no sistema nervoso central. Há estudos na literatura mostrando que o material particulado (MP) em suspensão na poluição do ar é uma ameaça a saúde, podendo afetar o cérebro além de outros órgãos, e causar alterações moleculares e bioquímicas que promovem a piora de crises epilépticas. Objetivo. Apresentar as principais evidências reportadas na literatura sobre os riscos da poluição do ar para a saúde, como afeta o sistema nervoso central, e possíveis riscos para pessoas com epilepsia. Método. Revisão não sistemática empregando as palavras-chaves "Poluição do ar", "material particulado", "Epilepsia" e "Doença neurodegenerativa". Resultados. Foram utilizados 24 manuscritos científicos, em inglês e português, para a confecção deste artigo. Conclusão. A poluição do ar representa um fator de risco para o aumento de crises em pacientes com epilepsia pelo fato de ativar mecanismos que modulam a excitabilidade neuronal (neuroinflamação) levando a uma piora na expressão das crises.
\end{abstract}

Unitermos. Poluição do ar; material particulado; epilepsia; doença neurodegenerativa

\begin{abstract}
Introduction. Epilepsy is considered a neurological disease that affects more than 50 million people worldwide. It is characterized by the manifestation of epileptic seizures, which are events with transient, self-limited activity, with defined signs and symptoms, and can be expressed in various ways depending on the area of onset in the central nervous system. There are many studies in the literature showing that particulate matter (PM) suspended in air pollution is harmful to health, affecting the brain in addition to other organs, and causing molecular and biochemical changes that promote the worsening of epileptic seizures. Objective. To present the main evidence reported in the literature on the health risks of air pollution, how it affects the central nervous system, and possible risks for people with epilepsy. Method. Non-systematic review using the keywords "Air pollution", "particulate matter", "Epilepsy" and "Neurodegenerative disease". Results. We used 24 scientific reports to prepare this review, in English and Portuguese, and neuroinflammation was one of the mechanisms associated with worsening of seizures. Conclusion. Air pollution can be a risk factor for increased seizures in patients with epilepsy because it activates mechanisms that modulate neuronal excitability (neuroinflammation) leading to a worsening in the expression of seizures. Keywords. Air pollution; particulate matter; epilepy; neurodegenerative disease
\end{abstract}

\section{Resumen}

Introducción. La epilepsia se considera una enfermedad neurológica que afecta a más de 50 millones de personas en todo el mundo. Se caracteriza por la manifestación de crisis epilépticas, que son eventos con actividad transitoria, autolimitada, con signos y síntomas definidos, y pueden expresarse de diversas formas dependiendo del área de inicio en el sistema 
nervioso central. Existen estudios en la literatura que muestran que el material particulado (PM) suspendido en la contaminación del aire es una amenaza para la salud, que afecta al cerebro además de otros órganos y provoca cambios moleculares y bioquímicos que promueven el empeoramiento de los ataques epilépticos. Objetivo. Presentar las principales evidencias reportadas en la literatura sobre los riesgos para la salud de la contaminación del aire, cómo afecta el sistema nervioso central y los posibles riesgos para las personas con epilepsia. Método. Revisión no sistemática utilizando las palabras clave "Contaminación atmosférica", "material particulado", "Epilepsia" y "Enfermedad neurodegenerativa". Resultados. Para la elaboración de este artículo se utilizaron 24 manuscritos científicos, en inglés y portugués. Conclusión. La contaminación del aire representa un factor de riesgo para el aumento de convulsiones en pacientes con epilepsia porque activa mecanismos que modulan la excitabilidad neuronal (neuroinflamación) conduciendo a un empeoramiento en la expresión de las convulsiones.

Palabras clave. Contaminación del aire; material particulado; epilepsia; enfermedad neurodegenerativa

Trabalho desenvolvido para o curso "Neurociência em Pauta" ("Neuroscience at hand"), ministrado pelo Programa de Pós-Graduação em Neurologia/Neurociências da Escola Paulista de Medicina, Unifesp, São Paulo-SP, Brasil.

\section{INTRODUÇÃO}

Como resultado do acelerado desenvolvimento industrial e urbanização, as taxas de poluição do ar aumentaram para níveis prejudiciais à saúde, o que constitui um problema global, sendo mais grave nas regiões mais desenvolvidas e populosas como África, Ásia ou Oriente Médio, que respiram altos níveis de poluição em comparação com outros países do mundo ${ }^{1,2}$.

Vários são os compostos que se acumulam no ambiente atmosférico, dos quais são monitorados aqueles que geram impacto na saúde como: ozônio, dióxido de enxofre, monóxido de carbono, dióxido de nitrogênio, chumbo e material particulado (MP). Existem outros contaminantes perigosos que incluem produtos químicos altamente neurotóxicos, como compostos orgânicos voláteis, formaldeído, tri e tetracloroetileno, benzeno, tolueno, 
hidrocarbonetos aromáticos policíclicos e metais como chumbo, manganês, ferro, mercúrio, arsênio, cádmio e cobalto ${ }^{3}$.

Um dos principais contaminantes ambientais com potencial efeito adverso à saúde de animais e humanos é o MP, formado por partículas derivadas de fonte primária da combustão de carvão, gasolina e óleo diesel ${ }^{4}$, associada às atividades humanas na área industrial, mineração, agricultura, tráfego de veículos e queima de combustíveis. Sua composição é variável, pois depende de fatores como o clima, a hora do dia, a estação do ano, a região, entre outros e pode estar presente na atmosfera no estado líquido ou sólido, com elementos biológicos, como pólen, bactérias, vírus e esporos ou elementos sólidos não biológicos em suspensão, como poeira e fumaça ${ }^{5}$.

O MP é classificado de acordo ao seu tamanho em partículas grossas, que possuem um diâmetro entre 2,5 e 10 micrômetros $\left(\mathrm{MP}_{10}\right)$, partículas finas que têm um diâmetro entre 0,1 e 2,5 ( $\left.\mathrm{MP}_{2,5}\right)$ micrômetros e partículas ultrafinas que têm um diâmetro menor ou igual a 0,1 micrômetros $\left(\mathrm{MP}_{0,1}\right)^{6}$. O MP é um dos principais problemas de saúde pública nas grandes cidades cujo transporte é feito por veículos com motor a queima de diesel; é um agente agressor silencioso, ao qual muitas pessoas que vivem nesses ambientes estão constantemente expostas.

Nesta revisão, vamos analisar as evidências existentes sobre o impacto da poluição do ar no corpo, como afeta o 
sistema nervoso central, e porque representa riscos para pessoas com epilepsia.

\section{Efeitos sistêmicos da exposição à poluição do ar}

Partículas finas e ultrafinas podem entrar no corpo diretamente ao serem inaladas, atingindo o bulbo olfatório e os pulmões, ou indiretamente quando ingeridas e entram na corrente sanguínea7. O MP10 se deposita na via aérea proximal e geralmente essas partículas são expelidas pela varredura mucociliar. As partículas menores (MP2.5 e MP0.1) conseguem atingir as vias aéreas pequenas e distais, onde entram em contato com a corrente sanguínea podendo atingir órgãos mais distais como os rins, o coração e até o sistema nervoso central ${ }^{4,6}$.

A deposição de MP2,5 nos bronquíolos e alvéolos leva à ruptura da barreira epitelial das vias aéreas, causa alteração das vias de sinalização celular, prejuízo na fagocitose, estresse oxidativo e inflamação. A presença do MP0,1 na circulação pode causar disfunção da fibrinólise com subsequente resposta inflamatória celular em tecidos não pulmonares ${ }^{4,6}$. Ao nível renal, por exemplo, produz uma resposta inflamatória e oxidativa, com lesão vascular (endotelial) $^{4,6}$. No coração, aumenta a suscetibilidade a arritmias, com prejuízo da função autonômica cardíaca e repolarização $0^{4,6}$.

Vasoconstrição, disfunção endotelial e aumento dos níveis de espécies reativas de oxigênio (ROS) ocorrem nos vasos sanguíneos, e em geral no nível sistêmico pode-se 
observar inflamação e estresse oxidativo, que se caracteriza por aumento da resposta inflamatória celular, com maior número de plaquetas e leucócitos circulantes, aumento da expressão de citocinas como IL-6, IL-8, IL-1 $\beta$, TNF e ativação de vias geradoras de ROS ${ }^{4,6}$.

\section{Efeitos da poluição no sistema nervoso central}

Cérebro em desenvolvimento

O cérebro em desenvolvimento é muito mais sensível às agressões ambientais do que o cérebro adulto. Danos tóxicos podem interferir em processos importantes do desenvolvimento cerebral como proliferação e migração neuronal, neurogênese, alteração na função de células gliais, entre outros, podendo ser permanentes ${ }^{8}$. Da mesma forma, a exposição ao ar poluído pode ter efeitos deletérios no cérebro, tanto nos estágios embrionários quanto pós-natais. De acordo com estudos da Organização Mundial de Saúde (OMS), a exposição começa na fase fetal e é cumulativa ao longo da vida².

Durante a fase fetal, a exposição causa alteração da função placentária que está relacionada ao desenvolvimento do corpo caloso, diminui o transporte transplacentário de oxigênio, restringe o crescimento fetal e também induz inflamação placentária e sistêmica ${ }^{9}$. Alguns autores reportam que a exposição à partículas finas $(M P 2,5)$ estaria relacionada com alterações estruturais do córtex cerebral do lobo frontal, occipital e parietal, apresentando diminuição da espessura ${ }^{10}$. Igualmente, exposição à altas concentrações de 
MP estão associadas ao estreitamento da substância cinzenta na área do lobo frontal e occipital ${ }^{11}$.

A exposição à poluentes ambientais no período pósnatal não afeta tanto a estrutura cerebral, porém pode prejudicar a maturação neurocognitiva da criança. Há estudos mostrando alterações cerebrais funcionais com diminuição na conectividade neuronal entre os lobos frontal e parietal, e aumento na atividade cerebral em repouso, entre as áreas motoras e os lobos pré-frontal e parietal, bem como as diferentes regiões do lobo frontal com o lobo parietal e o córtex cingulado ${ }^{11}$ após exposição ao ar poluído pela queima de diesel por tráfego veicular.

Efeitos da poluição no cérebro adulto

O cérebro adulto também está propenso aos danos causados pelo MP. A inflamação sistêmica no organismo pode causar ruptura da barreira hematoencefálica tornandoa permeável à partículas finas e ultrafinas, desencadeando uma resposta inflamatória cerebral constante que altera as respostas inibitórias e excitatórias, por meio diminuição na síntese de GABA ou aumento na concentração de IL-1B como resultado da ativação não controlada da resposta imune contra a neuroinflamação ${ }^{12}$.

Estudos têm evidenciado a presença de inflamação sistêmica em resposta à exposição da poluição do ar, além da neuroinflamação, estresse oxidativo e dano cerebrovascular. Modelos experimentais e cultura celular 
mostram ativação da micróglia e de astrócitos após exposição à nanopartículas ou ozônio ${ }^{13}$.

Todas essas alterações têm impacto negativo nas atividades da vida diária. Conforme citado, a exposição ao MP2,5 está associada ao baixo desempenho em testes cognitivos, de memória e de orientação em adultos com mais de 55 anos, e o efeito é cumulativo, ou seja, essas pessoas foram expostas por longo tempo ao ar poluído. Estudos realizados em adultos jovens não mostram o mesmo impacto nesses parâmetros cognitivos ${ }^{5}$. Além do impacto negativo sobre a função de aprendizado e memória, as alterações causadas pela exposição prolongada ao MP podem aumentar a excitabilidade neuronal causando prejuízo ao paciente com epilepsia, por exemplo, que pode ter aumento de suas crises em função das altas taxas de poluição.

\section{Poluição do ar e epilepsia}

Poluição e riscos para pessoas com epilepsia

Vários estudos de na literatura, mostraram uma correlação positiva entre altas taxas de MP e aumento de ocorrência de crises em pacientes com epilepsia, porém poucos autores investigaram os mecanismos envolvidos com a hiperexcitabilidade ${ }^{14-17}$.

A epilepsia é considerada uma doença neurológica, crônica e não transmissível, que afeta mais de 50 milhões de pessoas no mundo. Aproximadamente $80 \%$ dos casos são diagnosticados em países de baixa renda. As crises epilépticas são manifestações de sinais e sintomas que 
refletem atividade neuronal excessiva e síncrona em redes cerebrais. A Liga Internacional Contra Epilepsia (ILAE) define epilepsia pela presença de uma das seguintes condições: apresentar pelo menos duas crises não provocadas (ou reflexas) com intervalo maior a 24 horas; apresentar uma crise não provocada (ou reflexa) com probabilidade da ocorrência de crises subsequentes semelhante ao risco de recorrência geral (pelo menos 60\%) após duas crises não provocadas, ocorrendo nos próximos 10 anos; ou ainda ter o diagnóstico de uma síndrome de epilepsia ${ }^{18}$.

As crises podem ser classificadas em focais, quando se iniciam em um dos hemisférios, generalizadas, quando se iniciam simultaneamente em ambos os hemisférios, ou de início desconhecido. O nível de consciência e as manifestações motoras auxiliam na classificação das crises ${ }^{19}$. Crises disperceptivas são aquelas que ocorrem com prejuízo da consciência e perceptivas quando há preservação da consciência $^{19}$.

As alterações fisiopatológicas envolvidas com as epilepsias têm sido extensivamente estudadas em modelos experimentais. A epileptogênese é o processo pelo qual o cérebro passa por alterações moleculares e estruturais que podem gerar crises espontâneas. As alterações moleculares que ocorrem durante a progressão da doença podem causar piora das crises, a fármaco resistência e comorbidades psiquiátricas $^{20}$.

Desequilíbrio entre a neurotransmissão inibitória GABAérgica e excitatória glutamatérgica, com propensão ao 
aumento de glutamato e redução de GABA é considerado o principal fator responsável pelo início das crises. 0 tratamento com medicamentos anti-crise (MAC) é a terapia de primeira escolha para tratar e prevenir crises, embora a resposta possa ser diferente de caso para caso. Apesar dos mais de 30 MACs disponíveis no mercado, aproximadamente $30 \%$ dos pacientes não atingem o controle adequado de suas crises, e se tornam fármaco resistentes. Nesses casos, outras terapias podem auxiliar no controle de crises como o uso de dietas (dieta cetogênica), estimulação cerebral ou ainda o procedimento cirúrgico para a retirada do foco epiléptico. São muitos os mecanismos que ocorrem durante a epileptogênese e que predispõem à hiperexcitabilidade, tais como alteração na circuitaria neurona e glial, rebrotamento de fibras musgosas no hipocampo, neurogênese e gliose 20 .

Ativação de astrócitos e micróglia podem contribuir para a hiperexcitabilidade de epileptogênese ${ }^{21-23}$. A micróglia pode ser ativada por vários fatores como citocinas circulantes e monócitos, ou por proteínas plasmáticas ou células do sistema imune que entram no cérebro quando a barreira hematoencefálica é quebrada, por exemplo durante o estado de mal epiléptico. Quando ativada, esta promove liberação de citocinas no SNC exacerbando o processo de neuroinflamação podendo causar morte neuronal ${ }^{20}$.

As citocinas IL1B e TNF-alfa, e outras moléculas liberadas pelos astrócitos e micróglias ativadas (HMGB1 e ATP), causam hiperexcitabilidade. Assim fatores que levam 
ao aumento de citocinas no cérebro são críticos pois pode aumentar a frequência de crises $^{20}$.

A associação entre epilepsia e poluição ambiental é relativamente recente. A pesquisa introdutória ao assunto, tornou evidente que exposição à poluição gasosa e às partículas finas, pode representar um fator de risco para pessoas com epilepsia em áreas urbanas no Chile. Cakmak et al ${ }^{14}$ mostraram pela primeira vez uma associação entre picos de poluição atmosférica e a elevada taxa de hospitalização por aumento de crises epiléticas. O aumento de crises foi associado aos poluentes $\mathrm{O}_{3}, \mathrm{~N}_{2} \mathrm{O}, \mathrm{SO}_{2}, \mathrm{CO}$, PM10 e PM2, $5^{14}$.

Seguindo um delineamento semelhante, um segundo estudo investigou a relação epilepsia e poluição em Xi'an, uma das maiores metrópoles com elevados níveis de poluição da China. Ao contrário do que se observou em 2010, somente os níveis de $\mathrm{N}_{2} \mathrm{O}$ e $\mathrm{SO}_{2}$ foram associados de forma positiva com o aumento de atendimentos ambulatoriais para epilepsia, e nesse estudo $\mathrm{O}_{3} \mathrm{O}_{3}$ teve atividade protetora contra as crises $^{15}$.

Uma pesquisa multicêntrica identificou 51.523 admissões hospitalar por epilepsia, em 47 hospitais de 10 cidades da China. Os dados indicaram que quando $\mathrm{N}_{2} \mathrm{O}, \mathrm{CO}$ e PM2,5 aumentaram acima dos níveis permitidos pela OMS, houve aumento de hospitalizações por crises convulsivas, mesmo em exposição a curto prazo ${ }^{16}$.

Chiang et al fizeram um estudo buscando correlacionar os efeitos da poluição e das condições meteorológicas na 
frequência de crises em Taiwan. No que se refere aos poluentes, os autores mostraram que 6 variáveis apresentaram correlação positiva com as crises epilépticas, o $\mathrm{NO}, \mathrm{CO}, \mathrm{N}_{2} \mathrm{O}, \mathrm{PM} 2,5, \mathrm{CHH} 4 \mathrm{E} \mathrm{NMHC}^{17}$.

Apesar de recente e das poucas evidências de trabalhos científicos, os estudos de meta-análise indicam que os países com índices elevados de poluição atmosférica têm uma associação positiva com aumento de crises, principalmente no inverno e em situações de exposição a curto prazo. Os mecanismos não são conhecidos, mas recente estudo mostrou aumento de citocinas IL-1B, IL-6 e TNF-alfa, associado a um desequilíbrio entre a neurotransmissão inibitória e excitatória, com um aumento na excitabilidade neuronal demonstrado pela abreviação na expressão de crises induzidas pelo PTZ, em ratos adultos que foram expostos ao MP2,5 durante o desenvolvimento ${ }^{24}$. Com base nesse estudo é possível sugerir que a poluição ativa cascatas de sinalização que podem aumentar a ocorrência de crises representando riscos para pacientes com epilepsia que vivem em grandes áreas urbanas industrializadas ou com muito tráfego de veículos com motor a queima de diesel.

\section{CONCLUSÃO}

A poluição do ar, especificamente 0 material particulado, tem um grande impacto negativo na saúde humana, e principalmente nos pacientes com epilepsia que podem ter suas crises aumentadas se forem expostos a altas taxas desses poluentes. A inflamação sistêmica causada 
pela poluição é um importante fator de risco para o desenvolvimento de doenças neurológicas, com efeito de curto e longo prazo, podendo causar hiperexcitabilidade neuronal.

O efeito danoso é proporcional ao tempo de exposição e todas as pessoas que vivem em grandes cidades industrializadas e com altas taxas de poluentes estão sujeitas aos riscos para a saúde. A poluição do ar é causada principalmente pela atividade humana em centros urbanos, como a queima de combustíveis fósseis nos automóveis. Todos podemos contribuir para reduzir esses poluentes, porém necessitamos de políticas públicas mais eficientes para minimizar os danos.

\section{REFERÊNCIAS}

1.Shi L, Yin Y, Zhang L, Wang S, Sillanpää M, Sun H. Design and engineering heterojunctions for the photoelectrochemical monitoring of environmental pollutants: A review. App Catal B Environm 2019;248:405-22. https://doi.org/10.1016/j.apcatb.2019.02.044

2. World Health Organization (WHO). Ambient AirPollution: A global assessment of exposure and burden of disease. World Health Organization. 2016,

https://apps. who.int/iris/handle/10665/250141

pp1-131.

3.Calderón-Garcidueñas $L$, Leray $E$, Heydarpour $P$, Torres-Jardón $R$, Reis J. Air pollution, a rising environmental risk fator for cognition, neuroinflammation and neurodegeneration: The clinical impact on children and beyond. Rev Neurologiq 2016;172:69-80. https://doi.org/10.1016/j.neurol.2015.10.008

4.Xu X, Nie S, Ding H, Hou F. Environmental pollution and kidney diseases. Nat Rev Nephrol 2018;14:313-24. https://doi.org/10.1038/nrneph.2018.11

5.Kilian J, Kitazawa M. The emerging risk of exposure to air pollution on cognitive decline and Alzheimer's disease - Evidence from epidemiological and animal studies. Biomed J 2018;41:414-62. https://doi.org/10.1016/j.bj.2018.06.001

6. World Health Organization (WHO). Health effects of particulate matter: Policy implications for countries in eastern Europe, Caucasus and central Asia. World Health Organization. 2013;1-15. 
https://www.euro.who.int/en/health-topics/environment-and-

health/air-quality/publications/2013/health-effects-of-particulatematter.-policy-implications-for-countries-in-eastern-europe,-

caucasus-and-central-asia-2013

7.Carletti C, Santos R, Sierra de Araújo L, Santos M, Veras M, Da Silva $R$, et al. Exposição pós-natal à poluentes do ar $(m p \leq 2,5)$ como fator de risco para o desenvolvimento de comportamentos de depressão e Ansiedade: um estudo experimental. Colloquium Vitae 2019;11:20-31. https://doi.org/10.5747/cv.2019.v11.n3.v267

8. Costa L, Cole T, Dao K, Chang Y, Garrick J. Developmental impact of air pollution on brain function. Neurochem Inter 2019;131:104580. https://doi.org/10.1016/j. neuint.2019.104580

9.Sunyer J, Dadvand P. Pre-natal brain development as a target for urban air pollution. Bas Clin Pharmacol Toxicol 2019;125:81-8. https://doi.org/10.1111/bcpt.13226

10.Guxens M, Lubczynska M, Muetzel R, Dalmau-Bueno A, Jaddoe V, Hoek G, et al. Air Pollution Exposure During Fetal Life, Brain Morphology, and Cognitive Function in School-Age Children. Bio Psychiatr 2018;84:295-303. https://doi.org/10.1016/j.biopsych.2018.01.016

11. Herting M, Younan D, Campbell C, Chen J. Outdoor Air Pollution and Brain Structure and Function From Across Childhood to Young Adulthood: A Methodological Review of Brain MRI Studies. Front Pub Health 2019;7:332. https://doi.org/10.3389/fpubh.2019.00332

12.Fernandes MJS, Carletti CO, Sierra de Araújo LF, Santos RC, Reis J. Respiratory gases, air pollution and epilepsy. Rev Neurologiq 2019;175:604-13. https://doi.org/10.1016/j.neurol.2019.07.013

13.Block ML, Calderón-Garcidueñas L. Air pollution: mechanisms of neuroinflammation and CNS disease. Trends Neurosci 2009;32:506516. https://doi.org/10.1016/j.tins.2009.05.009

14.Cakmak S, Dales RE, Vidal CB. Air pollution and hospitalization for epilepsy in Chile. Environ Inter 2010;36:501-5. https://doi.org/10.1016/j.envint.2010.03.008

15.Xu C, Fan Y-N, Kan H-D, Chen R-J, Liu J-H, Li Y-F, et al. The Novel Relationship between Urban Air Pollution and Epilepsy: A Time Series Study. PLoSONE 2016;11:e0161992.

https://doi.org/10.1371/journal.pone.0161992

16. Bao X, Tian X, Yang C, Li Y, Hu Y. Association between ambient air pollution and hospital admission for epilepsy in Eastern China. Epilep Res 2019;152:52-8.

https://doi.org/10.1016/j.eplepsyres.2019.02.012

17.Chiang K-L, Lee J-Y, Chang Y-M, Kuo F-C, Huang C-Y. The effect of weather, air pollution and seasonality on the number of patient visits for epileptic seizures: A population-based time-series study. Epilep Behav 2020;115:107487.

https://doi.org/10.1016/j.yebeh.2020.107487

18.Fisher RS, Acevedo C, Arzimanoglou A, Bogacz A, Cross JH, Elger $\mathrm{CE}$, et al. ILAE Official Report: A practical clinical definition of epilepsy. Epilepsia 2014;55:475-82. https://doi.org/10.1111/epi.12550 
19.Beghi E. The Epidemiology of Epilepsy. Neuroepidemiol 2020;54:185-91. https://doi.org/10.1159/000503831

20.Victor TR, Tsirka SE. Microglial contributions to aberrant neurogenesis and pathophysiology of epilepsy. Neuroimmunol Neuroinflamm 2020;7:234-47. https://doi.org/10.20517/23478659.2020 .02

21.Devinsky O, Vezzani A, Najjar S, De Lanerolle NC, Rogawski MA. Glia and epilepsy: excitability and inflammation. Trends Neurosci 2013;36:174-84. https://doi.org/10.1016/j.tins.2012.11.008

22. Eyo UB, Murugan $\mathrm{M}$, Wu LJ. Microglia-neuron communication in epilepsy. Glia 2016;65:5-18. https://doi.org/10.1002/glia.23006

23. Robel S, Buckingham SC, Boni JL, Campbell SL, Danbolt NC, Riedemann $\mathrm{T}$, et al. Reactive astrogliosis causes the development of spontaneous seizures. J Neurosci 2015;35:3330-45. https://doi.org/10.1523/JNEUROSCI.1574-14.2015

24. Carletti CO. Poluição do ar de São Paulo $(M P 2,5)$ como fator de risco para o desenvolvimento de distúrbios comportamentais e alterações moleculares no cérebro de ratos (Tese). São Paulo: Escola Paulista de Medicina, Universidade Federal de São Paulo, 2020, p163. 